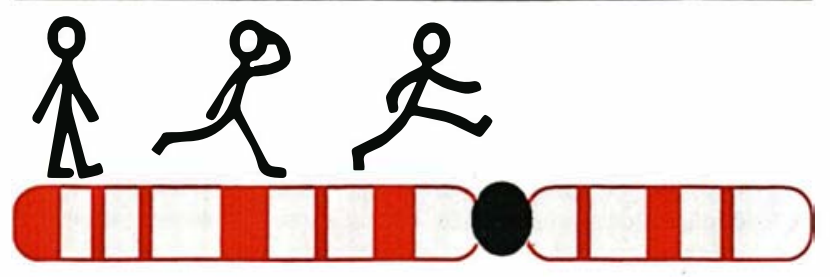

par Bertrand JORDAN

\title{
Génome français : de grandes espérances...
}

Une troisième place honorable L'examen de critères quantitatifs permet d'affirmer que notre pays se situe à la troisième place mondiale pour les recherches sur le génome humain. La figure 1 montre quelquesuns de ces indicateurs pour quatre nations (États-Unis, Japon, GrandeBretagne, France) en regard de leur produit national brut. Que l'on envisage, comme l'a fait la Fondation européenne de la science [1], le nombre de publications touchant à la cartographie des gènes chez l'homme, ou la proportion de nos compatriotes cooptés à HUGO avant que certains lobbies ne s'organisent, ou encore que l'on se livre à une analyse des communications présentées au congrès annuel du Génome à Cold Spring Harbor, la conclusion sera la même. Nous pouvons en tirer un certain réconfort puisque notre recherche se trouve ainsi placée devant celle du Japon ; d'un autre côté, le fait que notre production soit au moins deux fois plus faible que celle de la Grande-Bretagne incite à une grande modestie... En tout état de cause, la France fait partie des " poids lourds" dans ce secteur, ce qui lui donne à la fois des droits et des responsabilités.

Sur le plan qualitatif, l'évaluation est naturellement plus délicate. On peut noter quelques réussites récentes dans de très médiatiques " courses au gène " : localisation de l'amyotrophie spinale par l'équipe d'Arnold Munnich [2], clonage de la région de l'X fragile et analyse de sa variation par celle de Jean-Louis Mandel [3], et isolement du gène impliqué dans le syndrome de Kallman par le groupe de Christine Petit et Jean Weissen- que, dans chacun de ces cas, la compétition était très sévère, et que les publications françaises sont intervenues en même temps (à très peu de chose près, dans un sens ou dans l'autre) que celles d'une ou deux équipes étrangères travaillant sur le même sujet. De façon plus "structurelle ", la place de notre pays sur le plan international est due pour une bonne part à l'existence du CEPH et à la position centrale qu'il a su prendre dans l'établissement de la carte génétique humaine. Nous reviendrons plus loin sur cette organisation qui présente nombre de caractéristiques inhabituelles; rappelons simplement que Jean Dausset a su, dès 1980, prévoir que l'étude génétique de l'homme serait grandement facilitée par la constitution d'un " jeu " de familles dont l'ADN pourrait être fourni à de nombreux laboratoires et qui permettrait l'obtention d'un jeu cohérent de données. De fait, le CEPH a joué et continue de jouer un rôle primordial dans l'affinement de la carte génétique humaine, tout en s'étant investi dans plusieurs entreprises "génomiques ", en association avec une autre structure ad hoc, le Généthon.

Une remarque me paraît s'imposer à ce stade : le tissu scientifique français a été peu innovant sur le plan méthodologique, même s'il a su être créatif au niveau des structures. Si l'on passe en revue les grandes et petites révolutions conceptuelles et technologiques qui ont permis les progrès dans l'étude du génome, on verra que leur origine est presque toujours à l'étranger. Les concepts nouveaux - comme la cartographie génétique à l'aide des polymorphismes de l'ADN [5], le développement d'approches comme la génétique inverse - sont apparus outreAtlantique. Les outils nouveaux de la cartographie physique, ceux qui ont rendu possibles des cartes couvrant des chromosomes entiers, ont été eux aussi mis au point à l'étranger : gels pulsés [6], YAC [7], hybridation in situ non radioactive, exon trapping, proviennent pour l'essentiel des EtatsUnis; l'approche systématique par contigs de cosmides [8], les hybrides d'irradiation, de Grande-Bretagne ; quant au renouveau des méthodes de microdissection, son origine se situe pour l'essentiel en Allemagne [9]. La grande stabilité qui caractérise les structures de recherche françaises n'a donc pas été mise à profit pour investir à long terme dans les développements méthodologiques, et c'est à mon avis dommage. Cet état de fait tient sans doute à la faible estime dont est l'objet la technologie dans notre pays et dans nos instances d'évaluation. Quelles qu'en soient les raisons, nos laboratoires se sont limités à appliquer, parfois de façon très performante, les techniques mises au point ailleurs, mais n'ont que très rarement eu un rôle innovant à ce niveau.

\section{Un secteur associatif puissant} Contrairement à ce que l'on pense souvent, la recherche biologique française n'est pas le domaine réservé du "tout-État". Certes la place des laboratoires publics, Inserm, Cnrs, avec leurs forces et leurs faiblesses, est très importante. Mais l'initiative privée joue, et depuis longtemps, un rôle notable. Cela est particulièrement vrai pour le cancer avec les fondations comme la Ligue nationale française contre le cancer (LNFCC) ou 
l'Association de recherche sur le cancer (ARC) qui mobilisent des moyens considérables. Pour le génome, l'entité "incontournable " est l'Association française contre les myopathies. L'AFM existe depuis de nombreuses années, mais elle a été transformée à partir du milieu des années quatre-vingt par un président très dynamique et convaincu de l'importance de la recherche médicale. L'association a eu recours à des modes de financement jusque-là inhabituels dans notre pays, en particulier le fameux Téléthon, et a drainé par ce moyen, depuis 1987, des sommes impressionnantes : entre 200 et 300 millions de francs (MF) chaque année. Une partie de ce montant finance diverses aides aux malades, mais la fraction consacrée à la recherche est significative : entre 100 et $200 \mathrm{MF}$. Pour prendre la mesure de ce que représente une telle somme, il faut la mettre en regard du budget d'un organisme public, et analyser les différents postes que comporte ce dernier. Le secteur "sciences de la vie" du Cnrs, pour prendre cet exemple, dispose d'un budget annuel total de l'ordre de $2000 \mathrm{MF}$. Beau montant, mais il faut savoir que les dépenses de personnel (fonctionnaire, donc incompressible) représentent plus de $75 \%$ de ce total. Les $25 \%$ restants, soit $500 \mathrm{MF}$, couvrent l'ensemble des dépenses de fonctionnement, d'équipement, d'investissement immobilier... Comme il n'est guère possible de restreindre considérablement les dotations des laboratoires d'une année sur l'autre, cela signifie que la marge réellement disponible pour des actions nouvelles et volontaristes est de quelques dizaines de millions de francs pour l'ensemble des disciplines couvertes par ce secteur (les chiffres pour l'inserm sont similaires, avec un peu moins de dépenses de personnel). Les 100 MF injectés par l'AFM dans la recherche sur les maladies génétiques (en sus du fonctionnement du "Généthon " mentionné plus loin) pèsent donc très lourd en comparaison, et donnent à cette association un impact considérable.

Cet effet est d'autant plus sensible que les objectifs et l'état d'esprit des représentants d'une association de maladies diffèrent de ceux d'un res$m / s n^{\circ} 3$, vol. 8 , mars 92 ponsable d'organisme. A travers les 250 ou 300 MF du Téléthon, c'est une demande sociale qui s'exprime, et qui réclame que l'on fasse, vite, quelque chose pour lutter contre ces maladies et obtenir des résultats concrets. Il est bon pour les chercheurs d'être confrontés à cette saine impatience ; mais des distorsions sont possibles, et pas toujours faciles à éviter.

\section{Des modes d'organisation antinomi-} ques

Le monde de la recherche génomique française combine deux extrêmes. D'un côté des laboratoires " publics ", Inserm ou Cnrs en général ; il y en a peut-être une vingtaine qui comptent au niveau international. Ces structures sont emprisonnées dans le corset du service public: financements modestes, décisions lentes. Il faut, par exemple, compter seize mois au minimum entre le dépôt d'une demande de création d'unité Inserm toute ficelée, et son éventuelle création, sans aucune garantie sur les moyens en personnel et crédits qui lui seront accordés. Ces entités ont surtout fort peu d'autonomie (le laboratoire n'a pas la personnalité morale, et le directeur ne peut signer aucun contrat) et quasiment aucune possibilité de gérer avec efficacité leur personnel. Difficile, dans ces conditions, de construire des structures dynamiques, évolutives, capables de réagir " au quart de tour", de mettre l'accent sur un thème chaud et de réviser leurs priorités dès que nécessaire... On peut certes arriver à un fonctionnement acceptable, mais au prix de beaucoup d'énergie et de quelques tours de passe-passe. Autant d'efforts perdus pour la recherche : la créativité dépensée pour tenter d'embaucher un informaticien au prix du marché grâce à des cumuls souvent illégaux aurait mieux été employée à affiner une stratégie expérimentale. L'intervention de l'AFM, qui distribue assez largement contrats et bourses, met de l'huile dans les rouages (parfois même beaucoup d'huile) mais ne suffit pas à compenser les défauts du système.

De l'autre côté de la barrière, les structures très atypiques que sont le $\mathrm{CEPH}$ et le Généthon. Le CEPH, fondé au départ avec les fonds per- sonnels de Jean Dausset (cela mérite d'être signalé), est toujours une association loi de 1901 . Il est néanmoins financé en grande partie sur des fonds publics, avec une subvention du ministère de la Recherche d'une vingtaine de millions de francs; un montant à peu près équivalent est fourni par d'autres sources dont l'AFM, la CEE... Ses activités sont multiples, et il n'est pas toujours facile de faire la part de ce qui est du " service " (le criblage de la banque de YAC, la fourniture des ADN des familles...) et l'activité de recherche proprement dite, d'autant plus que le CEPH est lui-même imbriqué avec le Généthon (voir plus bas). Mais, globalement, on peut noter que cette structure d'une cinquantaine de personnes est largement financée, et que son fonctionnement s'apparente plus à celui d'une entreprise que d'un laboratoire public, surtout en ce qui concerne le point vital de la gestion du personnel. C'est à mon avis, on l'aura compris, une bonne chose ; et de ce point de vue le CEPH s'apparente à un de ces Genome Centers établis aux États-Unis. En revanche, il me semble que ces derniers, bien financés eux aussi (une cinquantaine de millions de francs par an, salaires compris), sont soumis à une évaluation plus rigoureuse, à un planning de résultats plus quantifié que ne l'est le CEPH.

Le Généthon, lui, est un résultat de la "saine impatience " mentionnée plus haut. Au départ, le choc ressenti par Bernard Barataud, président de l'AFM, en constatant que des étapes cruciales mais très laborieuses du processus qui mène au gène d'une maladie étaient réalisées, pour l'essentiel, de façon manuelle. Diagnostic partagé par Daniel Cohen (CEPH), et qui amena à la conception d'un centre spécialisé équipé de matériel lourd et susceptible d'être utilisé à tour de rôle par des équipes de recherche pour accélérer leur travail à certaines phases critiques. C'était le concept du Généthon, installé depuis maintenant plus d'un an à Évry, dans la banlieue sud de Paris. Important investissement, puisque l'AFM y consacre cette année un budget de $74 \mathrm{MF}$ (l'équipement est amorti sur trois ans) et y a recruté 130 personnes. 


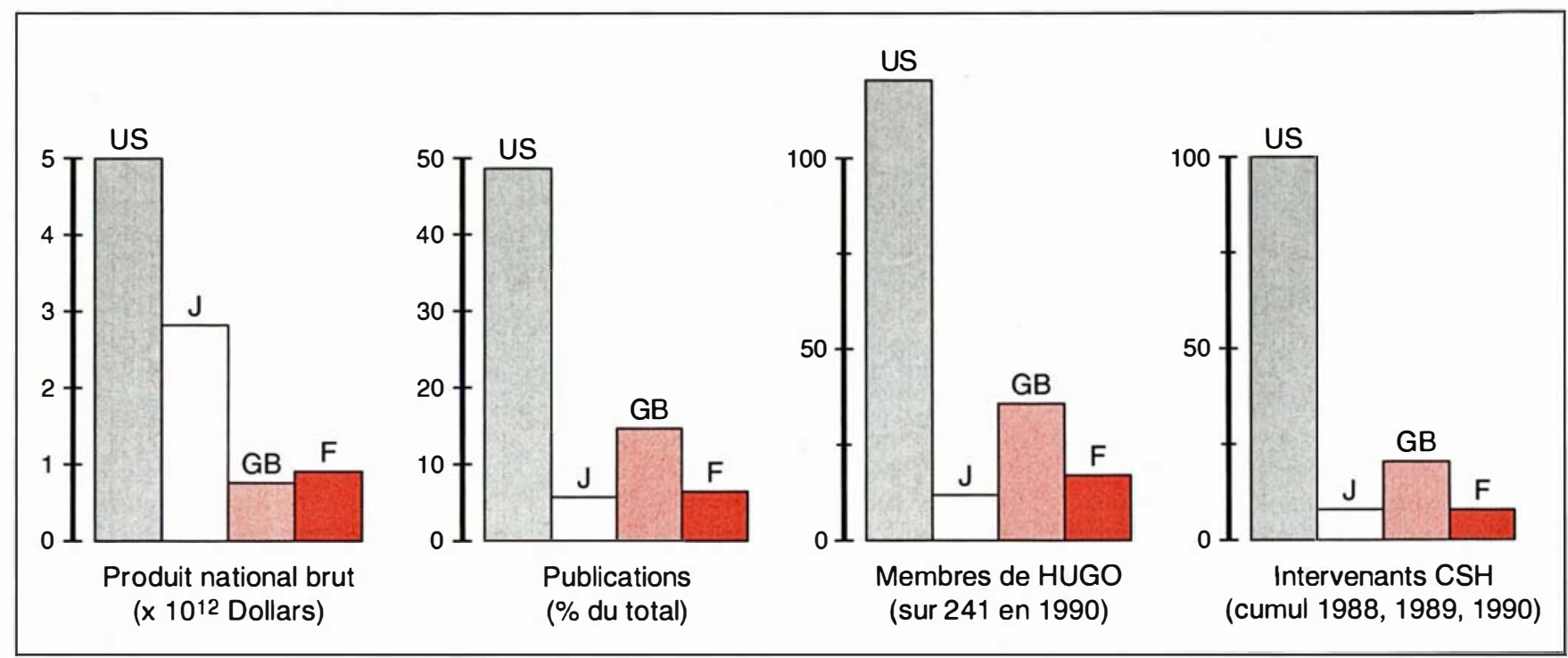

Figure 1. Cette figure présente quelques éléments de comparaison entre quatre grands pays. Premier panneau, à gauche, leur produit national brut en milliers de milliards de dollars; puis la production scientifique estimée par leur part des articles touchant au génome humain (étude réalisée pour la Fondation européenne de la science). Le troisième panneau montre le nombre de membres de l'organisation HUGO en mars 1990. Les campagnes de cooptation suivantes ont été marquées par l'apparition de lobbies efficaces dans certains pays comme l'Allemagne (plus de dix élus en une campagne), alors que d'autres pays s'en désintéressaient (un seul candidat français); enfin, le quatrième panneau montre le cumul des intervenants en session plénière au colloque annuel Genome mapping and sequencing de Cold Spring Harbor. On y remarque un "effet géographique " assez net...

L'idée est d'y rassembler un ensemble d'automates, par exemple vingt exemplaires de la machine à Southern blots conçue dans le cadre du programme LABIMAP* que viennent utiliser les équipes extérieures : plus d'une dizaine y ont eu effectivement recours. Le Généthon abrite aussi des projets comme celui de Charles Auffray sur le séquençage massif d'ADNc, de Daniel Cohen sur la cartographie physique à l'aide de YAC ou de Jean Weissenbach sur l'isolement de très nombreux " microsatellites " destinés à faire progresser la cartographie génétique.

Le monde du Génome français est donc, on le voit, très divers. Il est même un peu schizophrénique, ou en tous cas conflictuel, partagé qu'il est entre des laboratoires opérant dans un cadre très contraignant (bien plus qu'un Genome Center, pour reprendre ce point de comparaison) et d'autres

" Programme européen, dans le cadre "Eurêka", associant le CEPH, l'entreprise Bertin, la société Amersham... pour la mise au point d'une sére d'auto- assez débridés et disposant de moyens très supérieurs. C'est un terreau fertile pour le développement de jalousies inévitables entre équipes où les dotations (et même les salaires !) diffèrent considérablement. Ajoutons à cela que ce monde comporte plusieurs fortes personnalités dont la modération n'est pas la vertu première et qui ont une nette tendance à prendre toute critique pour une attaque personnelle, et l'on comprendra que le secteur n'est pas de tout repos et qu'il y règne une ambiance peu favorable à la sérénité.

Faut-il un programme Génome ? Puisque ce dernier n'a pas encore réellement démarré, on peut légitimement se poser la question. Après tout, nous sommes à la troisième place, les bonnes équipes trouvent sans trop de peine des contrats (grâce surtout à l'AFM)... pourquoi ne pas continuer comme cela ? A mon avis, un tel programme est indispensable, et vite. L'AFM, qui donne actuellement un ballon d'oxygène à ces recherches, a indiqué son intention de financer la génétique pendant trois ans (déjà bien entamés) mais de pas- ser ensuite au soutien des approches thérapeutiques (thérapie génique ou autres). C'est de toute façon un soutien aléatoire : qui peut assurer que l'intérêt du public se maintiendra, que le Téléthon continuera à "marcher ", qu'une autre cause ne sollicitera pas le public avec succès? Il faut donc organiser le relais, et donner aux laboratoires publics la souplesse nécessaire (en finances et en personnel) pour répondre aux défis du génome dans le cadre d'un programme planifié sur quelques années. Il faut aussi assurer l'avenir du CEPH (et du Généthon) en les faisant rentrer - dans une certaine mesure - dans un cadre plus " normal " mais, surtout, sans perdre les éléments positifs de leur structure. Un programme Génome français clairement annoncé, avec des structures transparentes, des moyens d'action et un financement assuré sur quelques années pourrait utilement restructurer ce monde dont j'ai décrit les contradictions, tout en impulsant les actions spécifiques indispensables (informatique, banques...). Il permettrait aussi qu'il y ait - enfin ! - un représentant de la France au niveau 
international. D'importantes négociations sont actuellement engagées : sur les banques de données et particulièrement celle de Baltimore, la Genome Data Bank, sur la brevetabilité des séquences... La participation de la France est désirée, attendue dans ces négociations ; mais, dans le flou actuel, personne ne peut réellement parler en son nom, et les occasions d'infléchir le cours des événements se perdent... Il y a donc urgence.

\section{Le difficile accouchement du GIP Génome}

Commençons par un peu d'histoire... Au printemps 1990 (deux ans déjà), le ministre de la Recherche chargeait Philippe Lazar, directeur de l'Inserm, de lui faire des propositions pour un programme Génome français. A l'époque, les États-Unis avaient investi dans ce secteur de façon significative (plus d'une centaine de millions de dollars par an), le Japon annonçait ses intentions, et la Grande-Bretagne avait effectivement démarré un an plus tôt son Human Gene Mapping Programme. Il y avait donc urgence... A son tour Philippe Lazar chargeait Philippe Kourilsky (Institut Pasteur) de rédiger un projet, ce que faisait ce dernier après une large consultation des scientifiques engagés dans ces recherches. Les propositions de Philippe Kourilsky recommandaient la mise sur pied d'un programme bénéficiant de crédits nouveaux de l'ordre de $100 \mathrm{MF}$ par an, privilégiant l'étude des gènes (donc des $\mathrm{ADNc}$ ) tout en soutenant informatique, organismes modèles et développements technologiques. Sur le plan organisationnel, il proposait la mise en place d'un "groupement d'intérêt public (GIP) permettant d'associer secteur public et privé et insistait sur la nécessité d'une création rapide de ce GIP, avec un directeur au profil de "manager ", ainsi que sur la nécessité d'une gestion souple du personnel. A l'automne 1990, le 17 octobre exactement, une conférence de presse du ministre annonçait le programme Génome français, selon des modalités assez proches du rapport de Philippe Kourilsky (à part la gestion du personnel) et avec un financement nouveau de $50 \mathrm{MF}$ pour 1991 et 100 pour $m / s n^{\circ} 3$, vol. 8 , mars 92
1992 [10]. Les choses semblaient donc bien parties.

La suite des événements allait démentir ces espérances. En mars 1991 (donc près de six mois après l'annonce ministérielle), Jacques Hanoune était chargé d'une mission exploratoire pour examiner comment ce GIP pourrait être mis sur pied. Des mois de négociations difficiles s'ensuivirent, au cours desquelles il apparut que les différents partenaires potentiels du GIP (organismes de recherche, associations, entreprises) avaient des idées très différentes sur ce qu'il devrait être et sur la répartition des sièges au conseil d'administration, que certains ne voyaient pas l'intérêt d'un GIP et qu'au niveau même du ministère, l'appui à ce projet n'était pas unanime... Au mois d'août, lors du onzième Human Gene Mapping Workshop à Londres, les choses n'avaient apparemment pas avancé. Quelques scientifiques français manifestèrent une inquiétude qui fut largement répercutée par la presse. Peu de temps après, le ministère s'attacha à répartir, dans des délais très courts, des contrats de recherche pour un montant total de plusieurs dizaines de millions de francs. Compte tenu de ces délais, la répartition fut faite dans des conditions acrobatiques et discutables (sans appel d'offres), suscitant des tensions supplémentaires dans un milieu qui n'en avait pas besoin. Mais ce fut ensuite au tour des heureux élus (ceux à qui l'on avait distribué de l'argent) d'être mécontents, car ces crédits qu'on leur avait octroyés n'existaient pas réellement ; plus précisément c'étaient des " autorisations de programme " et non des " crédits de paiement "(les initiés comprendront), et, pour les transformer en espèces sonnantes et trébuchantes, il fallait ponctionner l'Inserm, le Cnrs ou l'Inra... évidemment ravis du procédé...

Il semble maintenant que cette question soit en voie de règlement pour au moins une partie de ces crédits. Quant au programme Génome, il pourrait sous peu bénéficier d'un nouveau chargé de mission, un célèbre biologiste moléculaire de la levure qui vient de quitter la direction de son laboratoire de Gif-sur-Yvette.
Espérons que, cette fois, la tentative aboutira : il est grand temps...

\section{Bertrand Jordan}

Directeur de recherche au Cnrs, responsable du groupe de génétique moléculaire humaine. CIML, Inserm/Cnrs, case 906, 13288 Marseille Cedex 9, France.

\section{RÉFÉRENCES}

1. Academia Europaca. Research on the Human Genome in Europe and Its Relation to Activities Elsewhere in the World. Strasbourg : Academia Europaca, 1991.

2. Mclki J, Abdclhak S, Sheth P, et al. Gene for chronic proximal spinal muscular atrophies maps to chromosome 5q. Nature 1990 : $344: 767-8$

3. Heitz D, Rousseau F, Devys D, et al. Isolation of sequences that span the fragile $\mathrm{X}$ and identification of a fragile X-related $\mathrm{CpG}$ island. Science 1991; 251: 1236-39.

4. Legouis R, Hardelin JP, Levilliers J, et al. The candidate gene for the $\mathrm{X}$-linked Kallmann syndrome cncodes a protein related to adhesion molcculcs. Cell 1991; 67 : 423-35.

5. Botstein D, White RL, Skolnick $M$ Davis RW. Construction of a genetic linkage map in man using restriction fragment length polymorphisms. Am J Hum Genet $1980 ; 32$ : 314-31.

6. Schwartz DC, Cantor CR. Separation of yeast chromosome-sized DNAs by pulsed ficld gel clectrophoresis. Cell $1984 ; 37$ : 67-75.

7. Burke DT, Carle GF, Olson MF. Cloning of large segments of exogenous DNA into yeast artificial-chromosome vectors. Science $1987 ; 236$ : 806-8.

8. Coulson A, Sulston J, Brenner S, Karn J. Toward a physical map of the genome of the nematode Caenorhabditis elegans. Proc Natl Acad Sci USA 1986; 83 : 7821-5.

9. Ludecke HJ, Senger G, Claussen U, Horsthemke B. Cloning defined regions of the human genome by microdissection of banded chromosomes and enzymatic amplification. Nature $1989 ; 338$ : 348-50.

10. Jordan BR. Le programme français Génome humain. médecine/sciences $1990 ; 9$ : 908.

\section{TIRÉS A PART}

B. Jordan. 